\title{
¿Qué beneficios aporta el pinzamiento tardío del cordón umbilical frente al precoz en recién nacidos a término?
}

\author{
Rocío PÉREZ SILVESTRE \\ al316140@uji.es \\ Águeda Cervera Gasch \\ cerveraa@uji.es
}

\section{Resumen}

Introducción: El momento óptimo del pinzamiento del cordón umbilical causa controversia en las políticas sanitarias, sin existir un acuerdo a nivel nacional ni europeo. Hasta un período temprano, los países occidentales han llevado a cabo las intervenciones en el nacimiento de forma rutinaria, sin dar la importancia que precisa la relación madre-hijo. Por otro lado, está el nacimiento desde una forma fisiológica, que se centra en dejar que este se desarrolle de la forma más natural posible, respetando las elecciones autónomas de la madre y el bienestar del recién nacido. Método: Se realiza una revisión de la evidencia científica sobre los beneficios del pinzamiento tardío en recién nacidos a término comparando con el precoz, en Medline y Cochrane desde el 2009, completando la búsqueda con recomendaciones de la OMS, AEP, Reduca y Anales de Pediatría. Resultados: Las ventajas en que coinciden los artículos son que el pinzamiento tardío aumenta los depósitos de hierro, hematocrito y ferritina. Algunos corroboran que hay una diferencia positiva sobre los recién nacidos que se sometieron al pinzamiento tardío hasta los seis meses de edad. Los efectos secundarios no tienen alta prevalencia, siendo policitemia e ictericia, que en ocasiones precisan tratamiento. Conclusiones: Hay evidencia de que los beneficios del pinzamiento tardío superan a los del precoz, disminuyendo los porcentajes totales de anemia y las transfusiones y favoreciendo que los recién nacidos de países en vías de desarrollo con pocos recursos puedan optar por unos niveles férricos óptimos. Siempre teniendo en cuenta las contraindicaciones posibles y los efectos secundarios.

Palabras clave: cordón umbilical, pinzamiento tardío, pinzamiento precoz, a término.

\footnotetext{
Abstract

Introduction: The optimal timing of umbilical cord clamping is a controversial matter in health policies, with national or European agreements. For a considerable time, Western countries have carried out interventions at birth on a routine basis, without attaching the importance that the mother-child relationship requires. There is also birth from a physiological form, which focuses on allowing this to develop in the most natural way possible
} 
by respecting the mother's autonomous choice and the newborn's welfare. Method: A review of the scientific evidence for the benefits of delayed clamping in at-term infants was performed compared to the early MEDLINE and Cochrane from 2009. The search was completed with who, AEP, REDUCA and annals of Paediatric recommendations. Results: The coinciding advantages found in the review indicated that delayed clamping increases iron, ferritin and haemotocrit deposits. Some confirm a positive difference in the newborns subjected to delay until the age of 6 months. Side effects appeared to have no high prevalence, and polycythaemia and jaundice sometimes required treatment. Conclusions: Evidence was found that delayed clamping benefits outweigh early clamping ones as the total percentages of anaemia and transfusions lowered. Encouragement is offered to developing countries with few resources as this practice allows them to opt for optimum iron levels, but possible contraindications and side effects must always be taken into account.

Keywords: umbilical cord, delayed clamping, at-term birth.

\section{Introducción}

El momento óptimo del pinzamiento del cordón umbilical causa controversia en las políticas sanitarias, sin existir un acuerdo a nivel nacional ni europeo, dado que los numerosos efectos beneficiosos o perjudiciales no disponen de evidencia científica suficiente. Hasta un período temprano, los países occidentales han llevado a cabo las intervenciones en el nacimiento de forma rutinaria, sin dar la importancia que precisa la relación madre-hijo. Durante los años 90 se medicalizó el proceso del parto de forma excesiva y un ejemplo es el tema a tratar: tras administrar fármacos uterotónicos y realizar la episiotomía, se procedía al pinzamiento y corte del cordón, para finalizar con la expulsión de la placenta; era entonces cuando se separaba de la madre. Por otro lado está el nacimiento desde una forma fisiológica y humanizada, que se centra en dejar que este se desarrolle de la forma más natural posible, siempre respetando las elecciones autónomas de la madre y la familia y el bienestar del recién nacido. Este modo de nacimiento que se empezó a considerar a partir del 2000 es en el que se evitan las intervenciones a no ser que haya una razón. Con ese cambio surge el tema a tratar, el pinzamiento tardío, dejando que la circulación fetal cese y hasta que la placenta se separa del útero.

Son estos los motivos por los que se han llevado a cabo estudios experimentales que prueban, mediante datos cuantitativos, los beneficios o las desventajas de cada tipo de pinzamiento tanto en el momento del nacimiento como a los cuatro o seis meses de este.

Según la OMS, se entiende como pinzamiento precoz el efectuado en los primeros 60 segundos tras el parto y el tardío es el que se realiza a partir de un minuto o hasta que las pulsaciones de cordón umbilical cesan (OMS, 2015). Hay numerosos estudios sobre las consecuencias de estas intervenciones para ambos, madre y recién nacido, y sobre aspectos como el peso, el test de Apgar, la ictericia o la morbilidad, pero la revisión se centra en las del recién nacido, valorando específicamente las diferencias que aporta sobre los niveles de hemoglobina, hematocrito y ferritina.

El feto contiene un volumen sanguíneo de alrededor de $70 \mathrm{ml} / \mathrm{kg}$ y la placenta $45 \mathrm{ml} / \mathrm{kg}$. Esto supone que, después del nacimiento, mediante el pinzamiento tardío haya un mayor intercambio sanguíneo de la circulación placentaria, consiguiendo que aproximadamente 20-35 ml/ 
$\mathrm{kg}$ de sangre se transfunda, lo que supone un $5 \%$ del volumen y de las células sanguíneas (Escarpa, 2012).

\section{Método}

Se ha realizado una revisión bibliográfica sobre los beneficios que aporta el pinzamiento tardío del cordón umbilical frente al precoz en nacidos a término. La búsqueda bibliográfica se ha realizado en dos bases de datos: Medline y Cochrane. Los criterios de inclusión en la selección de artículos son: nacidos entre la semana 37 y 42 por parto eutócico o distócico y artículos publicados en los últimos 7 años, excluyéndose los que trataban sobre recién nacidos prematuros. Tras la lectura de título y abstract, se han recuperado a texto completo los artículos que cumplían los criterios de inclusión. Se ha completado la búsqueda con apoyo bibliográfico y recomendaciones pertenecientes a la Organización Mundial de la Salud (OMS), Asociación Española de Pediatría (AEP), la revista Reduca y un artículo publicado en el 2013 en Anales de Pediatría.

\section{Resultados}

La mayor parte de los artículos seleccionados aportan datos sobre los beneficios del pinzamiento tardío del cordón umbilical, incluyendo también las contraindicaciones o los perjuicios que puede acarrear este proceso.

Según la OMS «se recomienda el pinzamiento y corte tardío del cordón umbilical en todos los nacimientos, al tiempo que se inician simultáneamente los cuidados básicos del recién nacido» (OMS, 2015), incluyendo esta técnica en los alumbramientos de mujeres con VIH. También hay ocasiones en que está contraindicado; según la OMS, «no se recomienda cuando este sufre hipoxia y deba ser trasladado de inmediato para su reanimación» (OMS, 2015).

La Asociación Española de Pediatría recoge en su artículo una revisión de dos metanálisis y estudios aleatorizados, ambos sobre lactantes de dos a seis meses. Los primeros se obtuvieron los siguientes resultados: mayor hematocrito en el grupo de pinzamiento tardío $(2,00-5,40 \%)$, mayor concentración de ferritina $(16,58-19,21)$, depósitos de hierro $(7,67-32,13)$ y una reducción del riesgo de anemia $(0,40-0,70)$. Se observó un incremento del riesgo de policitemia asintomática sin otros efectos. En los segundos estudios, también hubo un incremento de la hemoglobina significativo, pero se indicó un aumento del riesgo de ictericia que precisó tratamiento (Sánchez, 2009).

Un estudio prospectivo publicado en Anales de Pediatría (Rincón, 2014) dividió a los pacientes en tres grupos, dependiendo del tiempo de pinzamiento (véase la tabla 1).

Tabla 1

Grupos en función del tiempo de pinzamiento

\begin{tabular}{cc}
\hline Grupo & Tiempo de pinzamiento \\
\hline 1 & $<60 \mathrm{sec}$. \\
2 & $1<2 \mathrm{~min}$ \\
3 & $2<3 \mathrm{~min}$ \\
\hline
\end{tabular}


Se realizaron análisis en el momento del nacimiento y a las 8 horas. Se obtuvieron los siguientes resultados: los niveles de hemoglobina, hematocrito, ferritina y bilirrubina aumentaron significativamente en el grupo 3, pero también los casos de policitemia asintomática.

La Federación de Asociaciones de Matronas de España recoge que «la única ventaja del pinzamiento precoz es la disminución del tiempo de alumbramiento; en cambio se observa más anemia e hipovolemia en los recién nacidos. El pinzamiento tardío mejora la oxigenación cerebral, reduce la necesidad de trasfundir, disminuye las hemorragias intraventriculares y la sepsis; aunque también existe alguna contraindicación, como el crecimiento intrauterino retardado» (Ortega, 2009).

En la base de datos Cochrane un artículo que recoge 15 ensayos obtuvo los siguientes datos: los recién nacidos a los que se les intervino mediante el pinzamiento tardío tenían concentraciones de hemoglobina superior, persistiendo hasta los tres o seis meses, por lo tanto presentaban menor riesgo de anemia ferropénica. Pero también obtuvieron un pequeño riesgo adicional de ictericia que requería fototerapia (McDonald, 2013).

Sobre la búsqueda realizada en la base de datos Medline se refleja lo siguiente: un artículo (Nesheli, 2014) sobre un estudio ejecutado en 60 recién nacidos asignados aleatoriamente a grupos de pinzamiento precoz y tardío corroboró que, a los seis meses tras el parto, los niveles de hemoglobina, hematocrito y hierro sérico fueron significativamente superiores en los del grupo de pinzamiento tardío. Además, no registraron casos de policitemia, ictericia u otros efectos secundarios indeseables.

Otro artículo (Andersson, 2011) realizó un estudio con 400 recién nacidos para controlar el estado del hierro en lactantes de cuatro meses, asignados aleatoriamente a dos grupos (véase la tabla 2).

Tabla 2

Grupos en función del tiempo de pinzamiento

\begin{tabular}{cc}
\hline Tipo de pinzamiento & Tiempo de pinzamiento \\
\hline Precoz & $<10 \mathrm{sec}$. \\
Tardío & $>180 \mathrm{sec}$. \\
\hline
\end{tabular}

Los resultados fueron unas concentraciones de hemoglobina $45 \%$ superiores en el grupo del pinzamiento tardío, sin encontrar diferencias en los síntomas postnatales respiratorios, policitemia, hiperbilirrubinemia o que requirieran fototerapia.

\section{Discusión y conclusiones}

Los estudios citados anteriormente coinciden en los beneficios que se obtienen mediante el pinzamiento tardío, aunque algunos otros, como el realizado por McDonald y cols. (2013), aportan más información, asegurando que las concentraciones de hemoglobina perduran hasta los seis meses del lactante. Este aspecto se refuerza por el estudio que realizaron Andersson y cols. (2011), cuando los lactantes tenían cuatro meses, obteniendo unas concentraciones superiores a los del grupo de pinzamiento precoz. En cuanto a efectos adversos del pinzamiento tardío del cordón umbilical, solamente dos de los artículos seleccionados señalan que se 
registraron: en el estudio de Sánchez y cols. (2009) y en el artículo de McDonald y cols. (2013) expuestos posteriormente.

Además se deben tener en cuenta unas consideraciones: el pinzamiento tardío se ha de considerar un impacto beneficioso en países en vías de desarrollo donde la anemia es más común en las madres o en los que no se dispone de los recursos alimentarios necesarios para una correcta nutrición de recién nacido (Van Rheenen, 2014). Por lo que el pinzamiento tardío puede ser un factor decisivo en el óptimo desarrollo y en la supervivencia del recién nacido. Por otra parte, se aconseja considerar el pinzamiento precoz en situaciones o características tanto del niño como de la madre; por ejemplo, en el sufrimiento fetal, la asfixia y el retardo del crecimiento intrauterino o si la madre tiene sensibilidad al factor $\mathrm{Rh}$ o diabetes (Rabe, 2007).

Por lo tanto, como conclusión, podemos citar que los numerosos estudios realizados en el tema aseguran que la modificación de un tiempo tan disminuido como uno o dos minutos en el clampaje del cordón umbilical en recién nacidos a término pueden aumentar de forma notable las concentraciones de hemoglobina y las reservas de hierro en los recién nacidos (Ortega, 2009). Esto produce una disminución en los primeros meses de vida de anemia y, por lo tanto, también disminuye la administración exógena de hierro, las transfusiones sanguíneas, etc. Se cita en alguno de estos estudios que se observan efectos nocivos como policitemia o ictericia, por ello sería un punto en contra realizar el pinzamiento tardío si no se disponen de medios para tratar la ictericia o hacer un buen control (Sánchez, 2009).

\section{Referencias bibliográficas}

Andersson, O., Hellström-Westas, L., Andersson, D. y Domellöf, M. (2011). Effect of delayed versus early umbilical cord clamping on neonatal outcomes and iron status at 4 months: a randomised controlled trial. BMJ (Clinical Research Ed.), 343, d7157. http://doi. org/10.1136/bmj.d7157

Escarpa, M. M., (2012). Pinzamiento tardío vs pinzamiento precoz del cordón umbilical. Reduca (Enfermería, Fisioterapia y Podología), 4, 242-270.

McDonald, S. J., Middleton, P., Dowswell, T., y Morris, P. S. (2013). Effect of timing of umbilical cord clamping of term infants on maternal and neonatal outcomes. The Cochrane Database of Systematic Reviews, 2, CD004074. Doi: 10.1002/14651858.CD004074.pub3

Nesheli, H. M., Esmailzadeh, S., Haghshenas, M., Bijani, A. y Moghaddams, T. G. (2014). Effect of late vs early clamping of the umbilical cord (on haemoglobin level) in full-term neonates. The Journal of the Pakistan Medical Association, 64, 1303-1305.

OMS (2015). Momento óptimo de pinzamiento del cordón umbilical para prevenir la anemia

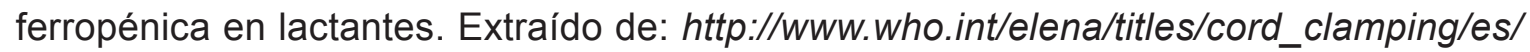

Ortega, E. M., Ruiz, A., Garrido, A. E. y Marchador, B. (2009). Evidencia científica en relación con el momento idóneo para pinzar el cordón umbilical. Matronas profesión, 10, 25-28.

Rabe, H., Reynolds, G. y Díaz-Rossello, J. (2007). Pinzamiento precoz versus pinzamiento tardío del cordón umbilical en prematuros. The Cochrane Database of Systematic Reviews, 4, CD003248. Doi: 10.1002/14651858.CD003248.pub2.

Rincón, D., Foguet, A., Rojas, M., Segarra, E., Sacristán, E., Teixidor, R. y cols. (2014). Tiempo de pinzamiento del cordón umbilical y complicaciones neonatales, un estudio prospectivo. Anales de Pediatría, 81, 142-148.

Sánchez, M., Pallás, C. R., Botet, F., Echániz, I., Castro, J. R., Narbona, E. y Comisión de Estándares de la Sociedad Española de Neonatología (2009). Recomendaciones para el 
cuidado y atención del recién nacido sano en el parto y en las primeras horas después del nacimiento. Anales de Pediatría, 71, 349-361.

Van Rheenen, P. y Brabin, B. J. (2004). Late umbilical cord-clamping as an intervention for reducing iron deficiency anaemia in term infants in developing and industrialised countries: a systematic review. Annals of Tropical Paediatrics, 24, 3-16. 